\title{
On the Expected Size of the 2D Visibility Complex
}

\author{
Hazel Everett* Sylvain Lazard* $\quad$ Sylvain Petitjean* $\quad$ Linqiao Zhang* ${ }^{* \dagger}$
}

\begin{abstract}
We study the expected size of the 2D visibility complex of randomly distributed objects in the plane. We prove that the asymptotic expected number of free bitangents (which correspond to 0 -faces of the visibility complex) among unit discs (or polygons of bounded aspect ratio and similar size) is linear and exhibit bounds in terms of the density of the objects. We also make an experimental assessment of the size of the visibility complex for disjoint random unit discs. We provide experimental estimates of the onset of the linear behavior and of the asymptotic slope and $y$-intercept of the number of free bitangents in terms of the density of discs. Finally, we analyze the quality of our estimates in terms of the density of discs.
\end{abstract}

\section{Introduction}

Visibility computations are central in computer graphics applications. Computing the limits of the umbra and penumbra cast by an area light source, identifying the set of blockers between any two polygons and determining the view from a given point are examples of visibility queries that are essential for the realistic rendering of 3D scenes. In global illumination algorithms, where the flow of light in a scene is simulated according to the laws of geometrical optics, visibility computations are excessively costly. In fact, more than half of the overall computation time can routinely be spent on visibility queries in radiosity simulations [12].

One approach to speeding up rendering is to store global visibility information in a data structure which can then be efficiently queried. The visibility complex, a partition of the set of maximal free line segments, and its 1-skeleton, the visibility skeleton, have been proposed as unified data structures encoding the visibility information of a scene [20] and have been used for rendering purposes $[6,8,9]$. Other related data structures include Pellegrini's ray-shooting structure [17], the aspect graph [18] and the visual hull [14]; see [7] for a recent survey.

One problem with these types of data structures which may prevent their application in practice is their potentially enormous size. In 3D, the size of the visibility complex of a set of $n$ triangles is $\Theta\left(n^{4}\right)$ in the worst case [9], which is prohibitive even for scenes of relatively modest size. Worst-case examples are somewhat artificial and indeed Durand et al. $[6,8]$ provided empirical evidence indicating that these worst-case upper bounds are largely pessimistic in practical situations; they observed a quadratic growth rate of the visibility skeleton, albeit for rather small scenes (with less than 1,500 triangles). The $\Theta\left(n^{2.5}\right)$ observed time complexity of their algorithm (which occasionally resorts to a systematic $\Theta\left(n^{5}\right)$ enumeration) and the lack of robustness of their implementation prevented experiments on much larger scenes. It was later proved that the expected size of the $3 \mathrm{D}$ visibility complex of random unit balls is linear [5]. Despite the fact that objects in graphics scenes are seldom distributed uniformly, the theoretical linear asymptotic bound hints that the experiments of Durand et al. may not have been performed for a sufficiently large number of objects to reach an asymptotic behavior. Because of the absence of a robust and efficient implementation for computing the visibility complex (or skeleton), estimating in practice the onset of the asymptotic linear behavior and the constants (slope and $y$-intercept) of the asymptote remains an open problem in 3D.

We focus here on the $2 \mathrm{D}$ case. While the worst-case complexity of the $2 \mathrm{D}$ visibility complex is quadratic, experimental results on scenes consisting of scattered triangles strongly suggest that the size of the visibility complex is linear [4]. In this paper, we carry out a detailed study of the size of the $2 \mathrm{D}$ visibility complex of discs and disc-like objects. First, we provide theoretical evidence to support the aforementioned observations. We prove that the expected

\footnotetext{
${ }^{*}$ LORIA - Université Nancy 2, INRIA Lorraine, and CNRS - Nancy, France. Firstname.Name@loria.fr.

$\dagger$ McGill University, Montréal, Canada. lzhang15@cs.mcgill.ca.
} 


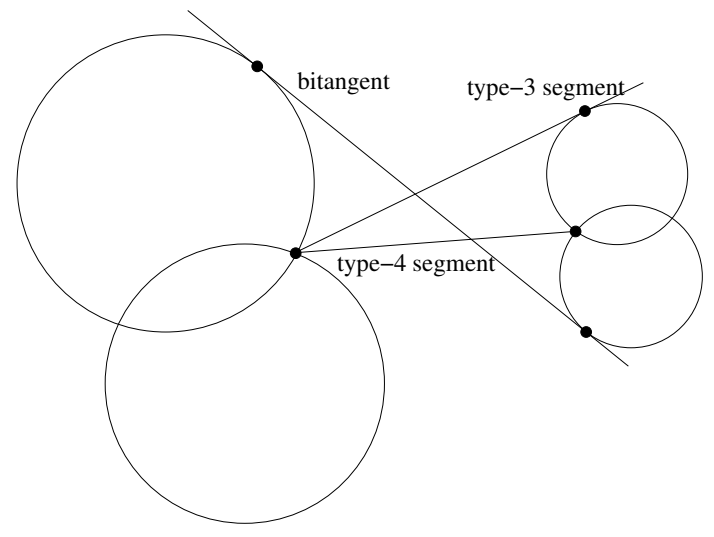

Figure 1: Bitangents and free segments corresponding to vertices of the visibility complex.

number of free bitangents, i.e., of maximal non-occluded line segments tangent to two discs, among $n$ uniformly distributed, possibly intersecting, unit discs in $\mathbb{R}^{2}$, is linear. This result is not surprising considering that the analog result was already proved in 3D for unit spheres [5]. We also show a linear bound on the expected number of maximal free line segments connecting two vertices of the union boundary of the set of discs (which we call type-4 free segments, as they are defined by four discs) or defined by one such vertex and tangent to another disc (which we call type-3 free segments); see Figure 1. These free segments are relevant since, together with the free bitangents, they correspond to the vertices of the visibility complex defined as the partition of the set of maximal free line segments in connected components of segments touching the same discs. Furthermore, we show that these bounds also hold for random bounded-complexity objects enclosed between discs of non-zero constant radii (whose 3D analog is not proved).

The main result of our paper is a detailed, theoretical and experimental, study of the constants in the asymptotic linear behavior of the expected number of free bitangents. We provide theoretical upper bounds and experimental estimates on the slope and $y$-intercept of the asymptote in terms of the density of discs. We also estimate the onset of the linear behavior in terms of the density.

The rest of this paper is organized as follows. Section 2 describes the models of distributions of unit discs we consider in this paper. We prove in Section 3 theoretical upper bounds on the expected number of free bitangents and free segments of types 3 and 4 among uniformly distributed, possibly intersecting, unit discs or polygons of bounded aspect ratio and similar size. We present in Section 4 our experiments and the interpolation of the number of free bitangents among random pairwise disjoint unit discs and conclude in Section 5.

\section{Models}

We describe in this section the two different probabilistic models we consider in this paper. The motivation for considering two different models comes from these simple observations:

- the theoretical analysis is most easily performed when the objects are independently chosen, and so can possibly intersect;

- the experimental assessment uses the only known released implementation of the $2 \mathrm{D}$ visibility complex that is time efficient (i.e., the one due to Angelier and Pocchiola [1]) and this implementation requires disjoint discs.

In what follows, let $n \in \mathbb{N}, D_{1}, \ldots, D_{n}$ be $n$ unit discs and call $p_{i}$ the center of $D_{i}$. Let also $\mathcal{U}$ (resp. $\mathcal{U}^{+}$) be the disc of radius $R>0$ (resp. $R+1$ ) centered at the origin $O$.

Intersecting-discs model. A sample scene in this model consists of $n$ unit discs $D_{i}, i=1, \ldots, n$, whose centers are independently chosen from the uniform distribution over the disc $\mathcal{U}$. Since the centers $p_{i}$ are distributed over $\mathcal{U}$, the discs $D_{i}$ may intersect each other and are contained in the universal disc $\mathcal{U}^{+}$. 

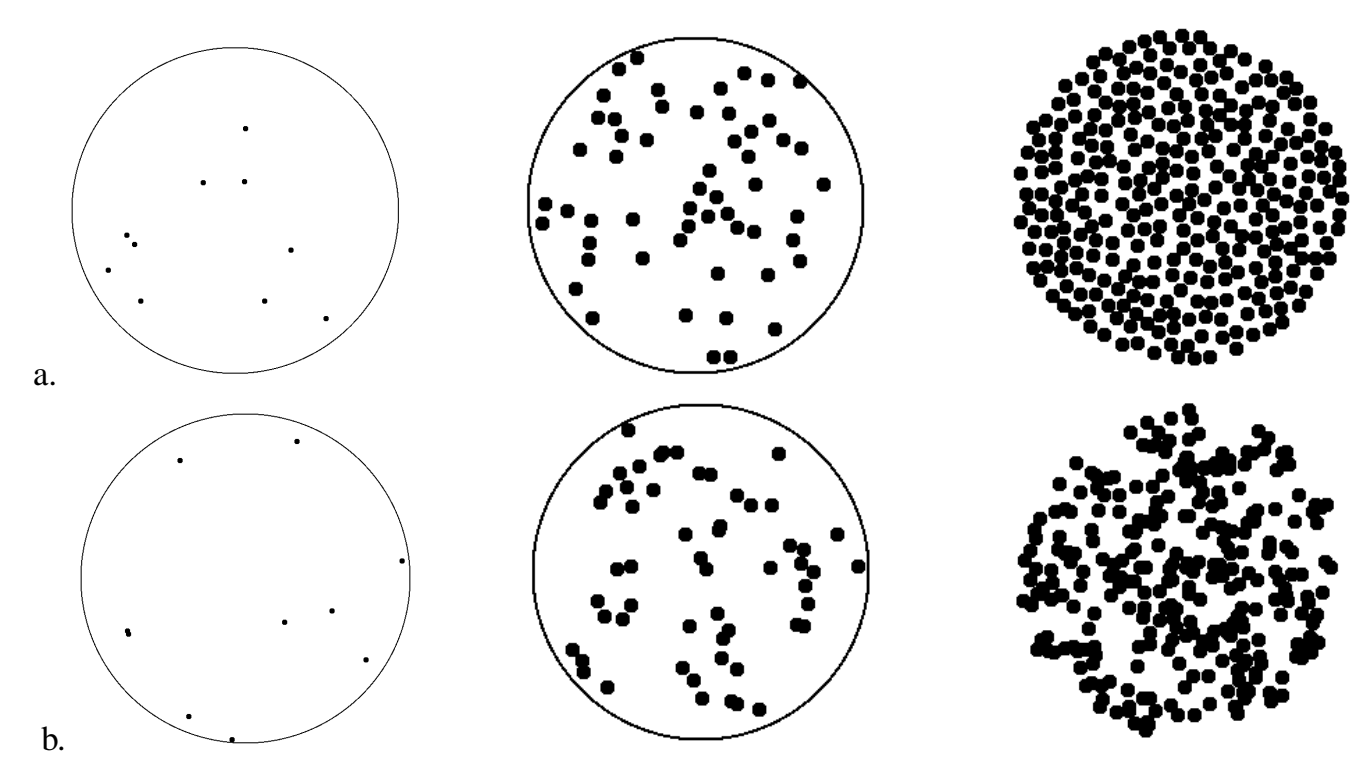

Figure 2: Scenes of (a) random disjoint and (b) possibly intersecting unit discs with densities $\mu=0.0025,0.1$, and 0.55 .

Note that random points over a disc of radius $R$ can be generated using two uniformly distributed variables $r \in$ $\left[0, R^{2}\right]$ and $\theta \in[0,2 \pi)$ and then taking

$$
\left\{\begin{array}{l}
x=\sqrt{r} \cos \theta \\
y=\sqrt{r} \sin \theta
\end{array}\right.
$$

The distribution induced by this model is uniform, in the sense that, for any region $A \subseteq \mathcal{U}$ of area $|A|$,

$$
\operatorname{Pr}((x, y) \in A)=\frac{|A|}{\pi R^{2}} .
$$

The average number of centers inside a unit disc inside $\mathcal{U}$ is thus $\mu=\frac{n}{R^{2}}$. The value $\mu$ reflects the "density" of points inside the universe. Since we are interested in asymptotic behavior as $n$ increases, we set $\mu$ to a constant value and define the radius $R$ of the universe $\mathcal{U}$ to be such that

$$
R^{2}=\frac{n}{\mu}
$$

Disjoint-discs model. The model we consider for our experiments is different from the theoretical one in the sense that we consider pairwise disjoint discs. A random sample is constructed by choosing the $n$ centers of discs one at a time from the uniform distribution over $\mathcal{U}$ with the constraint that each newly generated center is at distance larger than 2 from all the centers already generated.

Mimicking the intersecting-discs model, we set $\mu$ to be a constant and choose $R$ such that $R^{2}=\frac{n}{\mu}$. In this model, the density of discs inside $\mathcal{U}^{+}$(defined as the ratio of area covered by discs to the total area) is

$$
\frac{n}{(R+1)^{2}} \sim \mu \quad \text { when } \quad n \rightarrow \infty .
$$

Note that this distribution is different from the uniform distribution of disjoint discs which would be achieved by generating sets of $n$ centers independently from the uniform distribution over $\mathcal{U}$ until a set is generated in which all the corresponding discs are pairwise disjoint (such a distribution is clearly impractical for generating large and dense scenes).

In order to get a grasp on our two models, we present the results of some experiments. First, Figure 2 shows examples of random scenes for various densities for the two models. Figure 3 shows the percentage of free discs and 

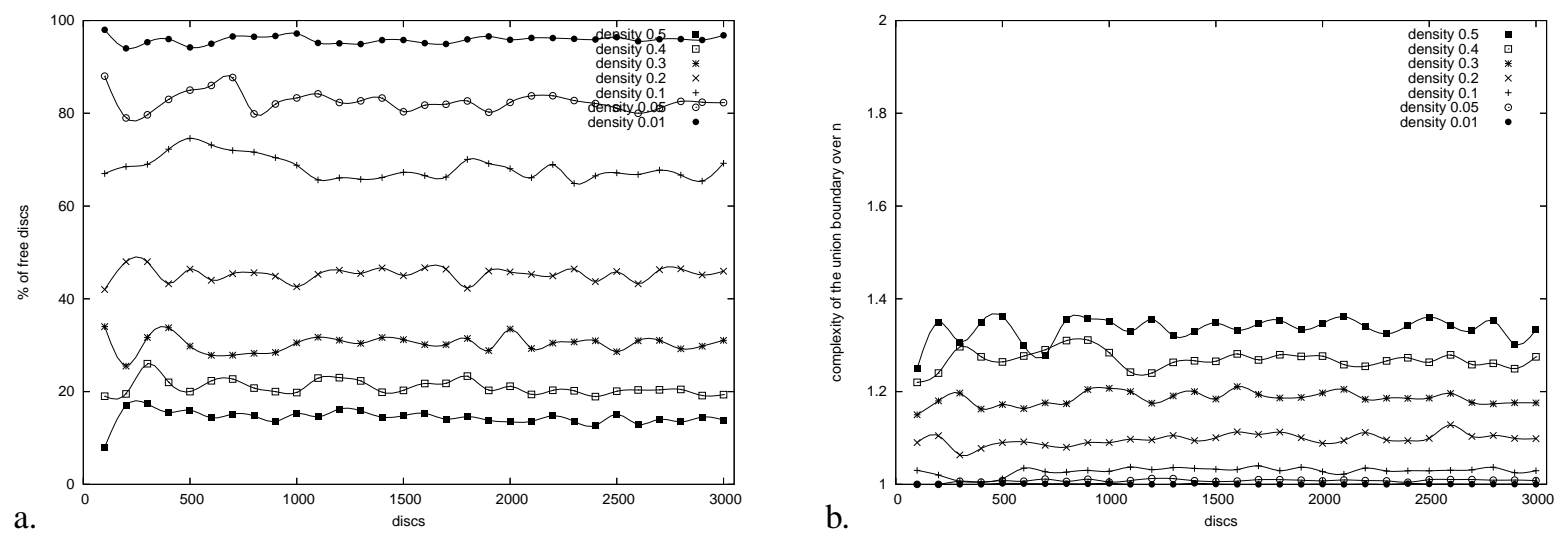

Figure 3: (a) Percentage of free discs in the intersecting-discs model. (b) Complexity of the union boundary over the number of discs.

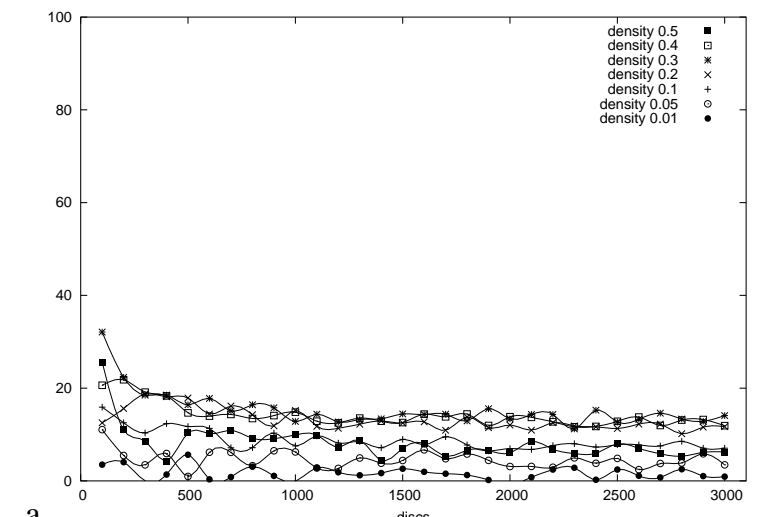

b.

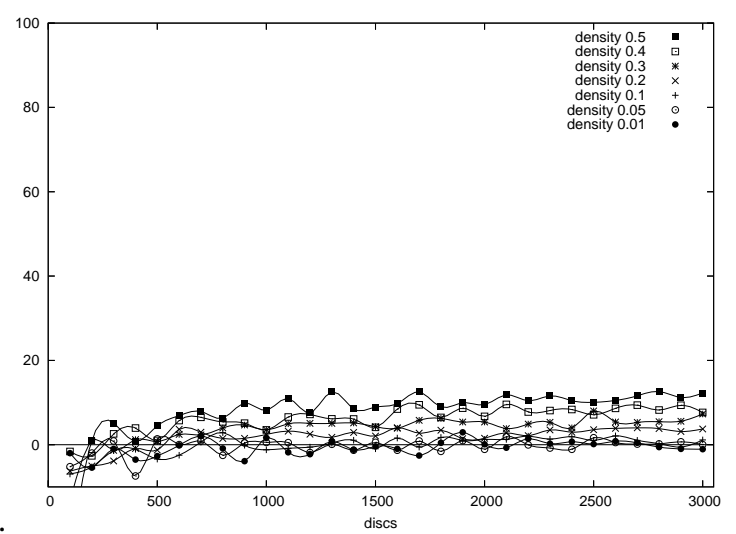

Figure 4: Discrepancy between the two models in terms of the number of free bitangents (expressed as a percentage): (a) $\frac{f-g}{f}$ where $f$ and $g$ are the number of free bitangents in the disjoint and intersecting-discs models, respectively; (b) $\frac{g^{\prime}-f}{g^{\prime}}$ where $g^{\prime}$ is the number of free bitangents plus type 3 and 4 free segments in the intersecting-discs model.

the complexity of the union boundary for random scenes in the intersecting-discs model. Notice that the percentage of free discs is substantial even for rather high densities but the complexity of the union boundary never exceeds $1.4 n$ for our data sets, which is consistent with the tight theoretical worst-case bound of $6 n-12$ [13]. Figure 4 shows that the discrepancy between the two models is rather small. First the number of free bitangents in the intersecting-discs model is asymptotically less by at most $15 \%$ than in the disjoint-discs model. Second, the number of free bitangents in the disjoint-discs model is asymptotically less by at most $12 \%$ than the number of vertices of the visibility complex, that is the number of free bitangents plus the number of type 3 and 4 free segments, in the intersecting-discs model. Finally, Figure 5 shows that the number of type 3 and 4 free segments does not exceed $40 \%$ of the total number of vertices of the visibility complex for the considered densities. Note finally that these graphs do not show any dependency on $n$ and that the standard deviations seem small (each data point corresponding to only one sample scene).

\section{Theoretical bound}

We prove in Section 3.1 a linear bound on the expected number of free bitangents of $n$ uniformly distributed discs. We then generalize the result, in Section 3.2, to free segments of types 3 and 4 and, in Section 3.3, to discs of various radii 


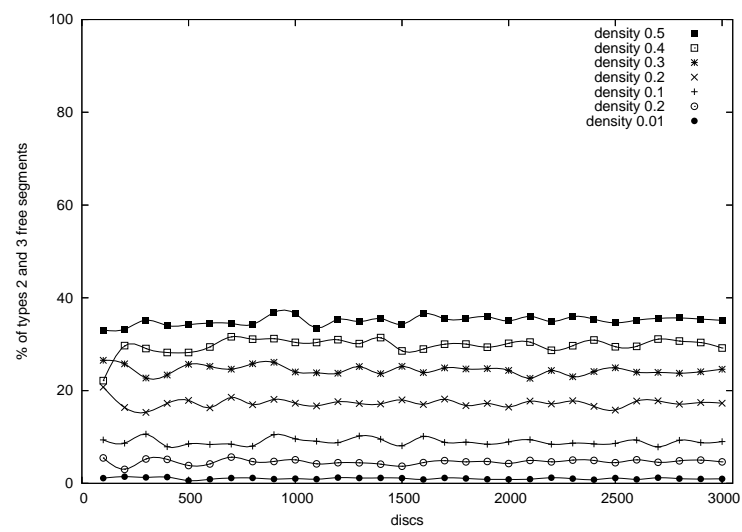

Figure 5: Percentage of the number of type 3 and 4 free segments over the total number of free bitangents and type 3 and 4 free segments, in the intersecting-discs model.

and polygons of bounded aspect ratio and similar size.

\subsection{Free bitangents}

We prove the following theorem.

Theorem 1. The expected number of free bitangents among $n$ uniformly distributed, possibly intersecting, unit discs is $\Theta(n)$. More precisely, the upper bound is less than

$$
8\left(\mu+\frac{4 \pi^{2}}{\mu}\right)(n-1) .
$$

Definitions. Let $\mathcal{N}$ be the set of ordered pairs $(i, j)$ chosen from $\{1,2, \ldots, n\}$ such that $i, j$ are distinct. In our model, the probability that two centers coincide is zero, so we may assume that any two discs admit at most 4 real common tangent lines. For any pair of discs we order arbitrarily the 4 bitangents (two of which are possibly complex) to the two discs.

Given two discs $D_{i}$ and $D_{j}$, we denote by $\mathcal{L}_{i, j}^{\omega}$, for $\omega$ in $\{1, \ldots, 4\}$, the event that the $\omega^{\text {th }}$ bitangent to $D_{i}$ and $D_{j}$ is real, and that $p_{i}$ is not closer than $p_{j}$ to the boundary of $\mathcal{U}$. Whenever $\mathcal{L}_{i, j}^{\omega}$ occurs, we denote the points of tangency of that line on $D_{i}$ and $D_{j}$ by $t_{i}^{\omega}$ and $t_{j}^{\omega}$, respectively. Let $\delta_{i, j}^{\omega}$ be the event that $\mathcal{L}_{i, j}^{\omega}$ occurs and the line segment $t_{i}^{\omega} t_{j}^{\omega}$ is not occluded.

Let $\mathbf{x}_{i, j}$ be the random variable representing the distance from $p_{i}$ to $p_{j}$, and $\mathbf{y}_{i}$ be the random variable representing the distance from $p_{i}$ to the boundary of the universe.

Proof of Theorem 1. There is a one-to-one correspondence between the free bitangents to $D_{i}$ and $D_{j}$ and the events $\delta_{i, j}^{\omega}$ that occur. We thus have the following straightforward lemma.

Lemma 2. The expected number of free bitangents among $n$ uniformly distributed unit discs is $\sum_{(i, j) \in \mathcal{N}} \sum_{\omega=1}^{4} \operatorname{Pr}\left(\delta_{i, j}^{\omega}\right)$.

We bound the probability $\operatorname{Pr}\left(\delta_{i, j}^{\omega}\right)$ by integrating over the distance $x$ between $p_{i}$ and $p_{j}$. However we treat independently the case where both $p_{i}$ and $p_{j}$ are close to the boundary of the universe. Dealing with boundary cases is usually the major difficulty with uniform distributions. However, handling the boundary case is here straightforward, since the expected number of centers falling in the annulus bounded by the circles of radius $R$ and $R-1$ is

$$
\left(R^{2}-(R-1)^{2}\right) \mu=(2 R-1) \mu=O(\sqrt{n}),
$$

so we trivially get that the expected number of bitangents between discs near the boundary is of order $n$. 
Lemma 3. $\operatorname{Pr}\left(\delta_{i, j}^{\omega}\right) \leqslant \frac{4 \mu}{n}+I$, where $I=\int_{x=0}^{2 R} \operatorname{Pr}\left(\delta_{i, j}^{\omega} \mid \mathbf{x}_{i, j}=x, \mathbf{y}_{i} \geqslant 1\right) \cdot \operatorname{Pr}\left(x \leqslant \mathbf{x}_{i, j}<x+\mathrm{d} x\right)$.

Proof. First notice that

$$
\operatorname{Pr}\left(\delta_{i, j}^{\omega}\right)=\operatorname{Pr}\left(\delta_{i, j}^{\omega} \cap\left(\mathbf{y}_{i}<1\right)\right)+\operatorname{Pr}\left(\delta_{i, j}^{\omega} \cap\left(\mathbf{y}_{i} \geqslant 1\right)\right) .
$$

Recall that if $\delta_{i, j}^{\omega}$ occurs then $p_{j}$ is closer to the boundary of $\mathcal{U}$ than $p_{i}$. $\operatorname{Thus} \operatorname{Pr}\left(\delta_{i, j}^{\omega} \cap\left(\mathbf{y}_{i}<1\right)\right)$ is less than or equal to the probability that both $p_{i}$ and $p_{j}$ lie within distance 1 of the boundary of $\mathcal{U}$. Since all the points are independently and identically drawn from the uniform distribution over $\mathcal{U}$, we have

$$
\operatorname{Pr}\left(\delta_{i, j}^{\omega} \cap\left(\mathbf{y}_{i}<1\right)\right) \leqslant \operatorname{Pr}\left(\mathbf{y}_{i}<1\right)^{2}=\left(\frac{\pi R^{2}-\pi(R-1)^{2}}{\pi R^{2}}\right)^{2}=\left(\frac{2 R-1}{R^{2}}\right)^{2} \leqslant \frac{4}{R^{2}}=\frac{4 \mu}{n} .
$$

Now, considering the second term of (1), we have

$$
\begin{aligned}
\operatorname{Pr}\left(\delta_{i, j}^{\omega} \cap\left(\mathbf{y}_{i} \geqslant 1\right)\right) & =\operatorname{Pr}\left(\delta_{i, j}^{\omega} \mid \mathbf{y}_{i} \geqslant 1\right) \cdot \operatorname{Pr}\left(\mathbf{y}_{i} \geqslant 1\right) \\
& \leqslant \operatorname{Pr}\left(\delta_{i, j}^{\omega} \mid \mathbf{y}_{i} \geqslant 1\right)=I
\end{aligned}
$$

by the Total Probability Theorem (see [16]).

We now prove that the integral $I$ is bounded by $O\left(\frac{1}{n}\right)$. For clarity, let $\Xi$ denote the event $\left(\mathbf{x}_{i, j}=x, \mathbf{y}_{i} \geqslant 1\right)$. In order to bound from above $\operatorname{Pr}\left(\delta_{i, j}^{\omega} \mid \Xi\right)$, we first need to bound from below the area of $\mathcal{H}_{i, j} \cap \mathcal{U}$, where $\mathcal{H}_{i, j}$ denotes the set of points at distance 1 or less to a tangent $t_{i}^{\omega} t_{j}^{\omega}$ corresponding to an event $\mathcal{L}_{i, j}^{\omega}$.

Lemma 4. When $\Xi$ and $\mathcal{L}_{i, j}^{\omega}$ occur, the area of $\mathcal{H}_{i, j} \cap \mathcal{U}$ is greater than $\frac{x}{2}$.

Proof. Let $K$ be the disc with diameter $p_{i} t_{i}^{\omega}$. Note that $K$ and $p_{j}$ are both contained in $\mathcal{U}$ and in $\mathcal{H}_{i, j}$. The convex hull of $p_{j}$ and $K$ is thus contained in $\mathcal{H}_{i, j} \cap \mathcal{U}$, and its area is half the area of the disc $K, \frac{\pi}{8}$, plus the area of a cone of apex $p_{j}$, of base a diameter of $K$, and of height greater than $x-\frac{1}{2}$. The area of that cone is at least $\frac{1}{2}\left(x-\frac{1}{2}\right)$, hence the area of $\mathcal{H}_{i, j} \cap \mathcal{U}$ is greater than $\frac{x}{2}+\frac{\pi}{8}-\frac{1}{4}>\frac{x}{2}$.

Lemma 5. $\operatorname{Pr}\left(\delta_{i, j}^{\omega} \mid \Xi\right)<2 \exp \left(-\frac{\mu x}{2 \pi}\right)$.

Proof. If $\delta_{i, j}^{\omega}$ occurs, then $\mathcal{L}_{i, j}^{\omega}$ necessarily occurs, thus

$$
\begin{aligned}
\operatorname{Pr}\left(\delta_{i, j}^{\omega} \mid \Xi\right) & =\operatorname{Pr}\left(\delta_{i, j}^{\omega} \cap \mathcal{L}_{i, j}^{\omega} \mid \Xi\right)=\operatorname{Pr}\left(\mathcal{L}_{i, j}^{\omega} \mid \Xi\right) \cdot \operatorname{Pr}\left(\delta_{i, j}^{\omega} \mid \mathcal{L}_{i, j}^{\omega}, \Xi\right) \\
& \leqslant \operatorname{Pr}\left(\delta_{i, j}^{\omega} \mid \mathcal{L}_{i, j}^{\omega}, \Xi\right) .
\end{aligned}
$$

$\operatorname{Pr}\left(\delta_{i, j}^{\omega} \mid \mathcal{L}_{i, j}^{\omega}\right)$ is equal to the probability that for all $\gamma \neq i, j$, point $p_{\gamma}$ is outside $\mathcal{H}_{i, j}$ given $\Xi$. Since all the points are independently and identically drawn from the uniform distribution over $\mathcal{U}$, we get

$$
\begin{aligned}
\operatorname{Pr}\left(\delta_{i, j}^{\omega} \mid \Xi\right) & \leqslant \operatorname{Pr}\left(p \notin \mathcal{H}_{i, j} \mid \mathcal{L}_{i, j}^{\omega}, \Xi\right)^{n-2} \\
& \leqslant\left(1-\left.\frac{\text { Area of } \mathcal{H}_{i, j} \cap \mathcal{U}}{\text { Area of } \mathcal{U}}\right|_{\mathcal{L}_{i, j}^{\omega}, \Xi}\right)^{n-2} .
\end{aligned}
$$

By Lemma 4, the area of $\mathcal{H}_{i, j} \cap \mathcal{U}$ is bounded from below by $\frac{x}{2}$, thus

$$
\operatorname{Pr}\left(\delta_{i, j}^{\omega} \mid \Xi\right)<\left(1-\frac{x}{2 \pi R^{2}}\right)^{n-2}
$$

with $x \leqslant 2 R$ and $R \geqslant 1$, since $\mathbf{y}_{i} \geqslant 1$. Thus $\frac{x}{2 \pi R^{2}} \leqslant \frac{1}{\pi R} \leqslant \frac{1}{\pi}$. For any $t, 1-t \leqslant e^{-t}$, thus for any $t \leqslant 1 / \pi$, we have $(1-t)^{n-2} \leqslant e^{-t n} e^{2 t}<2 e^{-t n}$. Hence

$$
\operatorname{Pr}\left(\delta_{i, j}^{\omega} \mid \Xi\right)<2 \exp \left(-\frac{x n}{2 \pi R^{2}}\right)=2 \exp \left(-\frac{\mu x}{2 \pi}\right) .
$$


We now bound the second term appearing in the integral $I$.

Lemma 6. $\operatorname{Pr}\left(x \leqslant \mathbf{x}_{i, j}<x+\mathrm{d} x\right) \leqslant \frac{2 x}{R^{2}} \mathrm{~d} x$.

Proof. When $p_{i}$ is given, $p_{j}$ must belong to a circular annulus between two circles of center $p_{i}$ and radii $x$ and $x+\mathrm{d} x$. The probability $\operatorname{Pr}\left(x \leqslant \mathbf{x}_{i, j}<x+\mathrm{d} x\right)$, if $p_{i}$ is known, is exactly the area of the part of the circular annulus inside $\mathcal{U}$ divided by the area of $\mathcal{U}$. The area of the part of the circular annulus inside $\mathcal{U}$ is bounded from above by the area of the circular annulus which is $2 \pi x \mathrm{~d} x$. Since the area of $\mathcal{U}$ is $\pi R^{2}$ we get the claimed bound. (The exact value of $\operatorname{Pr}\left(x \leqslant \mathbf{x}_{i, j}<x+\mathrm{d} x\right)$ is given in $[15,21]$ but the above approximate bound is enough for our purposes.)

We can now conclude by bounding the integral $I$.

Lemma 7. $I \leqslant \frac{16 \pi^{2}}{\mu n}$.

Proof. By Lemmas 5 and 6 we have

$$
I \leqslant \int_{x=0}^{2 R} 2 \exp \left(-\frac{\mu x}{2 \pi}\right) \cdot \frac{2 x}{R^{2}} \mathrm{~d} x \leqslant \frac{4 \mu}{n} \int_{x=0}^{+\infty} x \exp \left(-\frac{\mu x}{2 \pi}\right) \mathrm{d} x
$$

Changing $\frac{\mu x}{2 \pi}$ by $z$ we get

$$
I \leqslant \frac{4 \mu}{n} \int_{z=0}^{+\infty} \frac{2 \pi}{\mu} z \exp (-z) \frac{2 \pi}{\mu} d z \leqslant \frac{16 \pi^{2}}{\mu n}
$$

since $\int_{0}^{\infty} z \exp (-z) d z$ is bounded by 1 .

This completes the proof of the upper bound of Theorem 1 because Lemmas 2, 3, and 7 imply that the expected number of free bitangents is less than

$$
4\left(\begin{array}{l}
n \\
2
\end{array}\right)\left(\frac{4 \mu}{n}+\frac{16 \pi^{2}}{\mu n}\right)=8\left(\mu+\frac{4 \pi^{2}}{\mu}\right)(n-1) .
$$

Lemma 8. The expected number of free bitangents among $n$ uniformly distributed unit discs is in $\Omega(n)$.

Proof. Let $\delta_{i, j}$ be the event that the external (say, left) bitangent between discs $D_{i}$ and $D_{j}$ is not occluded. The probability that $\delta_{i, j}$ occurs is at least the probability that $\delta_{i, j}$ occurs and that the two discs centers $p_{i}$ and $p_{j}$ are greater than distance 1 from each other but less than distance 2 . Thus

$$
\operatorname{Pr}\left(\delta_{i, j}\right) \geqslant \operatorname{Pr}\left(1<\mathbf{x}_{i, j}<2\right) \cdot \operatorname{Pr}\left(\delta_{i, j} \mid\left(1<\mathbf{x}_{i, j}<2\right)\right) \text {. }
$$

For $n$ sufficiently large, the area of a circular annulus between two concentric circles of radii 1 and 2 centered in $\mathcal{U}$ is at least $\frac{3 \pi}{4}$, a quarter of the area of the annulus. Hence the probability that $p_{i}$ and $p_{j}$ are within distance 1 and 2 is at least $\frac{3 \pi}{4}$ divided by the area of $\mathcal{U}$, that is $\frac{3 \pi}{4} \cdot \frac{1}{\pi R^{2}}=\frac{3 \mu}{4 n}$.

$\operatorname{Pr}\left(\delta_{i, j} \mid\left(1<\mathbf{x}_{i, j}<2\right)\right)$ is the probability that the (left outer) bitangent to discs $D_{i}$ and $D_{j}$ is not occluded by $n-2$ other discs given that $p_{i}$ and $p_{j}$ are within distance 1 and 2. This probability is $\left(1-\frac{V}{\pi R^{2}}\right)^{n-2}$ where $V$ is the area of the region inside $\mathcal{U}$ and at distance at most 1 from the bitangent. Since $V \leqslant \pi+4$,

$$
\operatorname{Pr}\left(\delta_{i, j} \mid\left(1<\mathbf{x}_{i, j}<2\right)\right) \geqslant\left(1-\frac{\pi+4}{\pi R^{2}}\right)^{n-2}=\left(1-\frac{\left(1+\frac{4}{\pi}\right) \mu}{n}\right)^{n-2}>e^{-\left(1+\frac{4}{\pi}\right) \mu}
$$

We thus get that $\operatorname{Pr}\left(\delta_{i, j}\right) \geqslant \frac{3 \mu}{4 n} e^{-\left(1+\frac{4}{\pi}\right) \mu}$ and the result follows by Lemma 2 .

This completes the proof of Theorem 1 . 


\subsection{Free segments of types 3 and 4}

Theorem 1 generalizes in various ways.

Theorem 9. The expected number of free segments connecting two vertices of the union boundary of a set of $n$ uniformly distributed, possibly intersecting, unit discs is $\Theta(n)$. More precisely, the upper bound is less than

$$
11 \mu\left(\mu^{2}+8 \pi^{2}\right)(n-3)
$$

Proof. The proof of Theorem 1 generalizes as follows. We first define some notation similarly as before. Let $\mathcal{N}$ be the set of ordered pairs $(i, j, k, l)$ chosen from $\{1,2, \ldots, n\}$ such that $i, j, k, l$ are distinct. Given four discs $D_{i}, D_{j}, D_{k}$, and $D_{l}$, we denote by $\mathcal{L}_{i, j, k, l}^{\omega}$, for $\omega$ in $\{1, \ldots, 4\}$, the event that the $\omega^{\text {th }}$ segment joining an intersection point of $D_{i}$ and $D_{k}$ to an intersection point of $D_{j}$ and $D_{l}$ is real, that $p_{i}$ is the farthest of all four centers from the boundary of $\mathcal{U}$, and that $p_{j}$ is farther than $p_{l}$ to the boundary of $\mathcal{U}$. Whenever $\mathcal{L}_{i, j, k, l}^{\omega}$ occurs, we denote the endpoints of that segment by $t_{i}^{\omega}$ and $t_{j}^{\omega}$, respectively. Let $\delta_{i, j, k, l}^{\omega}$ be the event that $\mathcal{L}_{i, j, k, l}^{\omega}$ occurs and the line segment $t_{i}^{\omega} t_{j}^{\omega}$ is not occluded. Let $\mathbf{x}_{i, j}$ (resp. $\mathbf{x}_{i, k}, \mathbf{x}_{j, l}$ ) be the random variable representing the distance from $p_{i}$ to $p_{j}$ (resp. from $p_{i}$ to $p_{k}$ and from $p_{j}$ to $p_{l}$ ), and $\mathbf{y}_{i}$ (resp. $\mathbf{y}_{j}$ ) be the random variable representing the distance from $p_{i}$ (resp. $p_{j}$ ) to the boundary of the universe.

First, Lemma 2 generalizes directly to stating that the expected number of free segments of type 4 is $\sum_{(i, j, k, l) \in \mathcal{N}}$ $\sum_{\omega=1}^{4} \operatorname{Pr}\left(\delta_{i, j, k, l}^{\omega}\right)$. Second, Lemma 3 generalizes almost directly to

$$
\operatorname{Pr}\left(\delta_{i, j, k, l}^{\omega}\right) \leqslant\left(\frac{4 \mu}{n}\right)^{3}+\left(\frac{4 \mu}{n}\right)^{2} I
$$

where

$$
I=\int_{x=0}^{2 R} \operatorname{Pr}\left(\delta_{i, j}^{\omega} \mid \mathbf{x}_{i, j}=x, \mathbf{y}_{i} \geqslant 1, \mathbf{x}_{i, k} \leqslant 2, \mathbf{x}_{j, l} \leqslant 2\right) \cdot \operatorname{Pr}\left(x \leqslant \mathbf{x}_{i, j}<x+\mathrm{d} x\right),
$$

by noticing that

$$
\operatorname{Pr}\left(\delta_{i, j, k, l}^{\omega} \cap\left(\mathbf{y}_{i}<1\right)\right) \leqslant \operatorname{Pr}\left(\left(\mathbf{y}_{i}<1\right) \cap\left(\mathbf{y}_{j}<1\right) \cap\left(\mathbf{x}_{i, k} \leqslant 2\right) \cap\left(\mathbf{x}_{j, l} \leqslant 2\right)\right)=\operatorname{Pr}\left(\mathbf{y}_{i}<1\right)^{2} \cdot \operatorname{Pr}\left(\mathbf{x}_{i, k} \leqslant 2\right)^{2} \leqslant\left(\frac{4 \mu}{n}\right)^{3}
$$

since $\operatorname{Pr}\left(\mathbf{x}_{i, k} \leqslant 2\right) \leqslant \frac{\pi 2^{2}}{\pi R^{2}}=\frac{4 \mu}{n}$ and, as in the proof of Lemma $3, \operatorname{Pr}\left(\mathbf{y}_{i}<1\right)^{2} \leqslant \frac{4 \mu}{n}$. Lemma 4 generalizes directly. Lemma 5 generalizes after replacing $n-2$ by $n-4$, so that the constant in the upper bound becomes 4 instead of 2 . Therefore, the constant also doubles in Lemma 7. Hence, we get that the number of free segments of type 4 is at most

$$
4\left(\begin{array}{l}
n \\
4
\end{array}\right)\left(\left(\frac{4 \mu}{n}\right)^{3}+\left(\frac{4 \mu}{n}\right)^{2} \frac{32 \pi^{2}}{\mu n}\right) \leqslant \frac{32}{3} \mu\left(\mu^{2}+8 \pi^{2}\right)(n-3)
$$

which gives the upper bound. Finally, the proof of Lemma 8 also generalizes directly by noticing that

$$
\operatorname{Pr}\left(\delta_{i, j, k, l}\right) \geqslant \operatorname{Pr}\left(1<\mathbf{x}_{i, j}<2\right) \cdot \operatorname{Pr}\left(\mathbf{x}_{i, k} \leqslant 2\right) \cdot \operatorname{Pr}\left(\mathbf{x}_{j, l} \leqslant 2\right) \cdot \operatorname{Pr}\left(\delta_{i, j} \mid\left(1<\mathbf{x}_{i, j}<2\right),\left(\mathbf{x}_{i, k} \leqslant 2\right),\left(\mathbf{x}_{j, l} \leqslant 2\right)\right)
$$

which yields the linear lower bound.

Theorem 10. The expected number of free segments through an intersection point of the boundary of two discs and tangent to another disc among $n$ uniformly distributed, possibly intersecting, unit discs is $\Theta(n)$. More precisely, the upper bound is less than

$$
11\left(\mu^{2}+8 \pi^{2}\right)(n-2) \text {. }
$$

Proof. Similarly as in the proof of Theorem 9, we get that the expected number of free segments through an intersection point of the boundary of two discs and tangent to another disc is at most

$$
4\left(\begin{array}{l}
n \\
3
\end{array}\right)\left(\left(\frac{4 \mu}{n}\right)^{2}+\left(\frac{4 \mu}{n}\right) \frac{32 \pi^{2}}{\mu n}\right) \leqslant \frac{32}{3}\left(\mu^{2}+8 \pi^{2}\right)(n-2),
$$

which gives the upper bound. The lower bound follows from Lemma 8 similarly as in the proof of Theorem 9 . 


\subsection{Discs of various radii and polygons of bounded aspect ratio and similar size}

Theorems 1, 9 and 10 also generalize to other types of objects.

Theorem 11. The expected number of free bitangents and free segments of types 3 and 4 among $n$ discs or polygons of bounded complexity, each enclosed between two concentric discs of radii $r_{\text {min }}$ and $r_{\text {max }}$ whose centers are uniformly distributed in $\mathcal{U}$, is $\Theta(n)$.

Proof. The proof of Theorem 1 generalizes directly by considering the events $\mathbf{y}_{i} \geqslant r_{\text {max }}$ instead of $\mathbf{y}_{i} \geqslant 1$. The bounds in Lemmas 3, 4, 5, and 7 then become $\frac{4 \mu r_{\max }^{2}}{n}+I, \frac{x r_{\min }}{2}, 2 \exp \left(-\frac{\mu x r_{\min }}{2 \pi}\right)$, and $\frac{16 \pi^{2}}{\mu n r_{\min }^{2}}$, which yield an upper bound of $8\left(\mu r_{\max }^{2}+\frac{4 \pi^{2}}{\mu r_{\min }^{2}}\right)(n-1)$ on the number of free bitangents supported by two distinct objects. The linear upper bound on the number of free bitangents follows since the objects are of bounded complexity. The proof of the lower bound in Lemma 8 generalizes directly by considering the probability that $p_{i}$ and $p_{j}$ are within distance $r_{\min }+r_{\max }$ and $2\left(r_{\min }+r_{\text {max }}\right)$ instead of 1 and 2. The proofs of Theorems 9 and 10 also generalize by considering $\mathbf{x}_{i, k}$ and $\mathbf{x}_{j, l}$ less than $2 r_{\text {max }}$ instead of 2 . This leads to upper bounds of $11 \mu r_{\max }^{2}\left(\mu^{2} r_{\max }^{4}+\frac{8 \pi^{2}}{r_{\min }^{2}}\right)(n-3)$ on the number of free segments of type 4 and $11 r_{\max }^{2}\left(\mu^{2} r_{\max }^{2}+\frac{8 \pi^{2}}{r_{\min }^{2}}\right)(n-2)$ on the number of free segments of type 3 .

\section{Experiments}

We first describe our experiments in Section 4.1 and then present our experimental results and their interpretation in Section 4.2.

\subsection{Setting}

With the disjoint-discs model defined as in Section 2, we measure, for various densities, the number of bitangents in the scene. We also measure the memory usage and the running-time costs of computing these free bitangents.

We compute the visibility complex using a package due to Angelier and Pocchiola [1], based on the Greedy Flip Algorithm [2, 19], and the Simple_cartesian kernel and floating point (double) number type of CGAL [3].

We run experiments on scenes with up to 4,500 unit discs and density ranging from 0.0025 to 0.55 . We increment the density by 0.0025 for $\mu<0.025$ and by 0.025 for $\mu \geqslant 0.025$. We increment the number of discs by 40 up to 1,200 and by 100 after. For small and medium densities, i.e. $\mu \leqslant 0.01$ and $\mu \in[0.0125,0.0225]$, we compute the visibility complex for only up to 1,200 and 2,000 discs, respectively, because of memory limitations in the software implementation (see Section 5 for further discussion on this issue).

We do not consider densities $\mu$ larger than 0.55 because our scene generation scheme fails for such large densities. As Figure 2 shows, density 0.55 already implies a fairly dense scene. (Note that Thue proved in 1890 that the best packing of unit discs in the infinite plane is the regular hexagonal tiling - each disc being tangent to six others - and has density $\frac{\pi}{\sqrt{12}}$; thus $\frac{\pi}{\sqrt{12}} \approx 0.91$ is an upper bound for the density of our scenes.)

For each density value and number of discs we consider, we run 10 experiments and report the means of the measures. The standard deviations are very small and we do not report them. We report the number of oriented bitangents, the memory usage in units of $\mathrm{kBs}$ and the running time in units of $10^{-4}$ seconds (so that running time, number of bitangents and memory usage can be drawn on the same figure).

Note that the visibility complex package outputs oriented bitangents: for each maximal free non-oriented line segment tangent to two discs, the visibility complex implementation outputs two oriented bitangents. Since it is more intuitive to count non-oriented bitangents, we make the distinction between the two in what follows.

All the experiments were made on a 1686 machine with AMD Athlon $1.73 \mathrm{GHz}$ CPU running Linux and $1 \mathrm{~GB}$ of main memory. We use the getrusage() command to measure user time and mallinfo() function to measure memory usage. We made use of the ExpLab [10] environment to manage our experiments. 
a.
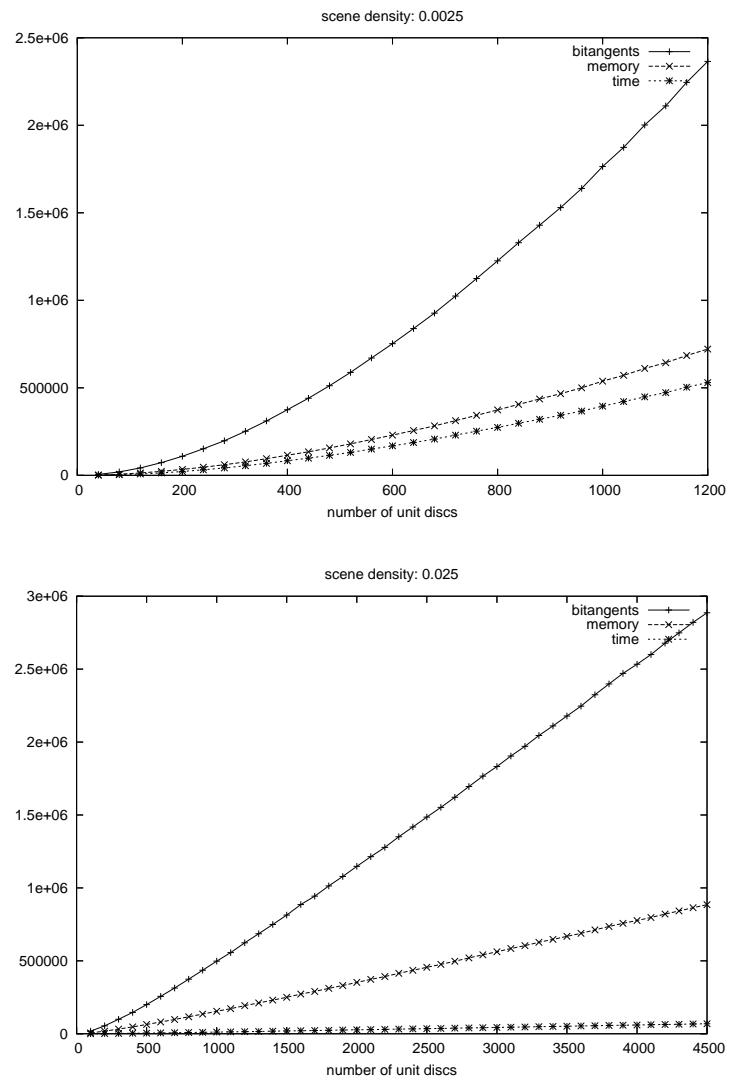

b.

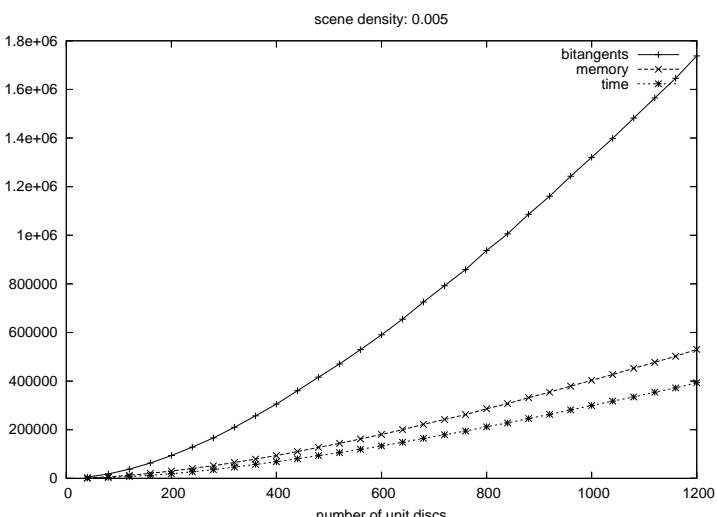

d.

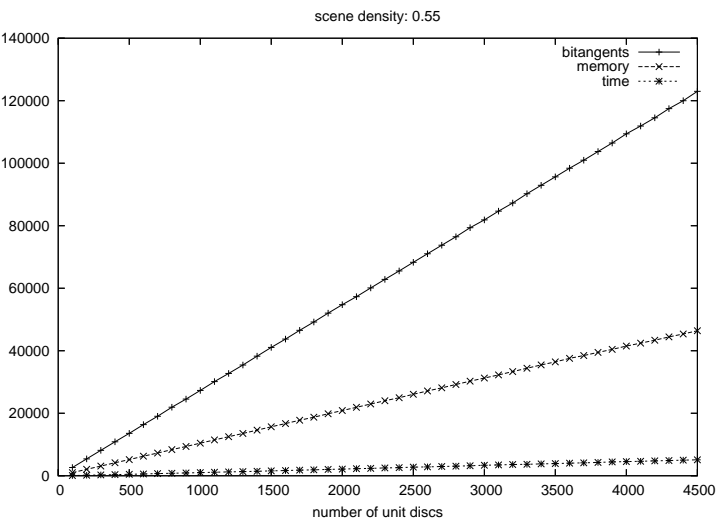

Figure 6: Plots of the number of oriented bitangents, memory usage, and running time in terms of the number of unit discs, when scene density is equal to (a) 0.0025 , (b) 0.005 , (c) 0.025 , and (d) 0.55 . The unit of the memory usage is $\mathrm{kBs}$, that of the running time is $10^{-4}$ seconds.

\subsection{Experimental results and interpretation}

We present here our experimental results. We display in Figure 6 the output of our experiments for four representative values of the density (equal to $0.0025,0.005,0.025$, and 0.55 ). Figure 6 shows quite clearly that the number of oriented bitangents, the memory usage, and the running time have a linear asymptotic behavior in terms of the number of discs ${ }^{1}$. We note that the slopes of the asymptotes are different for each density $\mu$ and are decreasing functions in terms of $\mu$. We also observe that the number of discs at which the linear behavior appears to start is a decreasing function of $\mu$.

In the rest of the section, we use least-squares fitting to estimate, in terms of scene density $\mu$ and number of discs $n$, the linear asymptote of the number of oriented bitangents and the onset of this linear behavior. For linear least-squares fitting on a set of $p$ data points $\left(x_{i}, y_{i}\right)$, recall that the correlation coefficient $r$, which measures the quality of fit, is defined as

$$
r=\frac{p \sum x_{i} y_{i}-\sum x_{i} \sum y_{i}}{\sqrt{\left(p \sum x_{i}^{2}-\left(\sum x_{i}\right)^{2}\right)\left(p \sum y_{i}^{2}-\left(\sum y_{i}\right)^{2}\right)}} .
$$

The closer $r$ is to 1 , the better the fit is.

\footnotetext{
${ }^{1}$ Note that the linear asymptotic behavior of the time complexity is only apparent since the time complexity of the Greedy Flip Algorithm is in $\Theta(n \log n+m)$ where $m$ is the size of the output. But for the values of $n$ we consider the $n \log n$ part is outweighed by the $m$ part.
} 

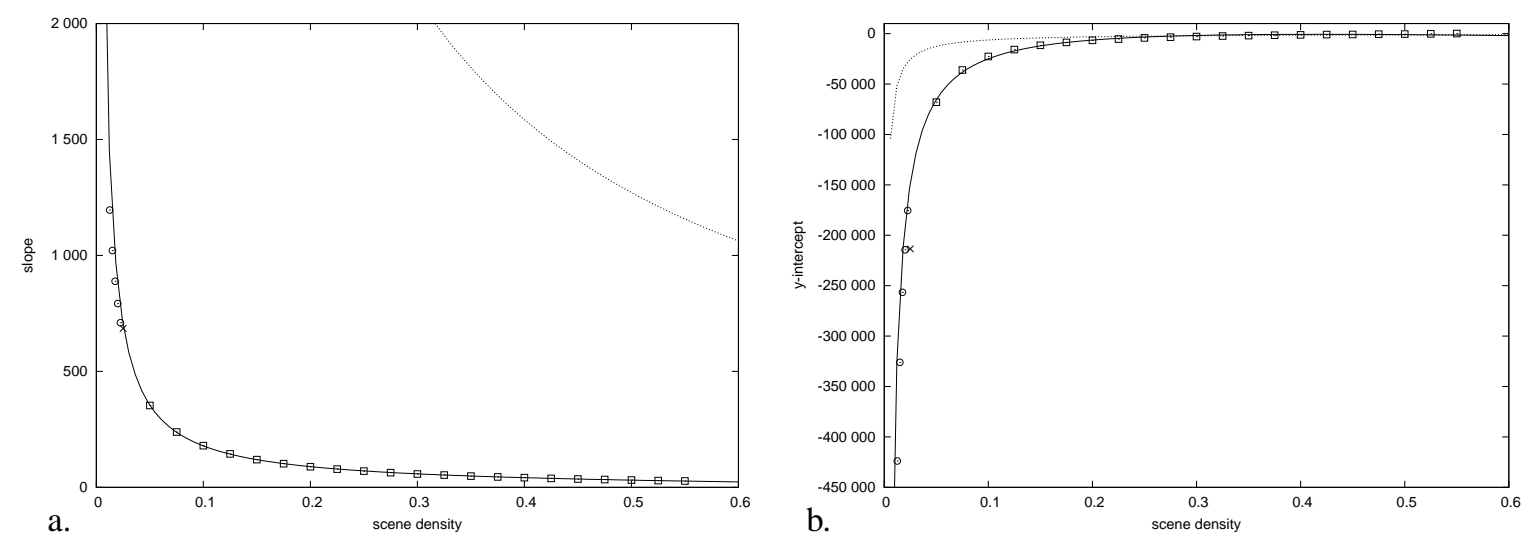

Figure 7: The (a) slope and (b) $y$-intercept, in terms of $\mu$, of the linear asymptote of the number of oriented bitangents (in terms of the number of discs): experimental data points and interpolations (of the square points) by (a) $\frac{17.49}{\mu}+$ $5.67-19.17 \mu$ and (b) $-\frac{4,182}{\mu}+19,255-23,789 \mu$. The dashed curves are the theoretical upper bounds of Theorem 1 (times two since the bitangents are here oriented).

\subsubsection{Asymptotic properties of the number of bitangents}

For each experimental density value $\mu \in[0.0025,0.55]$, we estimate the asymptote of the number of oriented bitangents (in terms of the number of discs) using a least-squares fitting on a subset of all the data points, as follows. We compute a least-squares fitting, first using all data points, and then recursively after removing the point corresponding to the smallest number of discs, until the correlation coefficient of the fit of the remaining set of points is larger than some threshold.

We choose the threshold for the correlation coefficient with care. Indeed, a threshold too small would imply that all the data points are always used for the least-squares fitting, which would not be satisfactory for small densities (see for instance Figure 6.a). A threshold too large would imply that only two data points are kept for the fitting which is also not satisfactory. In practice, we have a small window for a threshold that is neither too small nor too large. We choose the square of the threshold for the correlation coefficient to be equal to 0.99969 .

Figure 7 shows the estimated slopes and $y$-intercepts of the linear asymptotes for the scene densities that are larger or equal to 0.0125 in our experiments. We do not consider the asymptotes for smaller densities because they are not significant; indeed these asymptotes are only estimated by two points because of our choice of correlation-coefficient threshold.

We observe that the extracted slopes and $y$-intercepts appear intimately related to the inverse of $\mu$. Moreover, the slopes and $y$-intercepts are bounded theoretically (in a slightly different model where the discs may intersect) by functions of the type $\frac{a}{\mu}+b \mu-$ see Theorem 1 . We thus try to fit functions of the form $\frac{a}{\mu}+b \mu+c$ to the data points. However, we only interpolate the data points corresponding to densities strictly larger than 0.025 because we are only confident on the quality of the interpolated asymptotes for these densities. The reason for this is that when the density gets strictly smaller than 0.025 , the number of points used for estimating the asymptotes drops by more than half because the maximum number of discs used for the experiments drops from four thousand to two thousand, and the minimum number of discs used for interpolating the asymptotes increases to over 800 (see Figure 8); hence, for densities in $[0.0125,0.0225]$, the slopes and $y$-intercepts are thus estimated with fewer data points (namely between eight and twelve points). We also do not use the points of density 0.025 (the cross in Figure 7) because the $y$-intercept data point seems inaccurate. Note that although they are not used for interpolation, the estimated slopes and $y$-intercepts for $\mu \leqslant 0.025$ are used for asserting the quality of the fits.

Using least-squares fitting, we obtain the interpolating functions $\frac{17.49}{\mu}+5.67-19.17 \mu$ and $-\frac{4,182}{\mu}+19,255-$ $23,789 \mu$ for the slopes and $y$-intercepts respectively. As Figure 7 shows, the data points lie very close to the fitting curves. Moreover, the points corresponding to densities $\mu<0.025$ lie also quite close to the fitted curves, which is a good hint that our interpolations are satisfactory. 


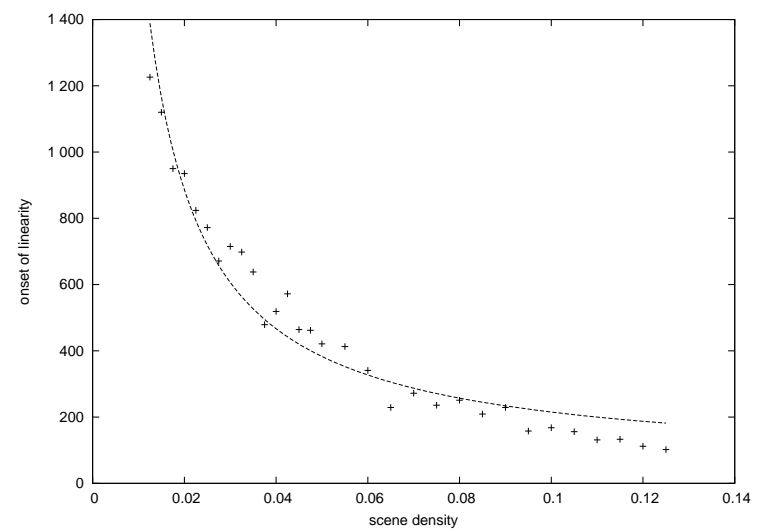

Figure 8: Onset of linearity in terms of the density $\mu$ : experimental data points and their fitting by $\frac{16.77}{\mu}+47.55$.

An interesting issue is to determine, as a function of $\mu$, the value $n_{0}$ of the number of discs at which the linear asymptotic behavior starts. We choose $n_{0}$ to be the smallest value of $n$ used for estimating the asymptote. Figure 8 shows the value of $n_{0}$ for densities in $[0.0125,0.125]$; note that we substantially refined the increment of the density for these experiments. We restricted ourselves to these densities because our data is only meaningful in that range in view of our choice of correlation-coefficient threshold. Indeed, outside of it, either only two points or all points are kept for estimating the asymptote.

Fitting these data points by a function of the form $\frac{a}{\mu}+b$, we obtain the function $\frac{16.77}{\mu}+47.55$. As Figure 8 shows, this interpolation is not nearly as good as for the slope and $y$-intercept of the asymptote. One of the reasons is that, for a fixed value of the density $\mu$, the number of bitangents has not been computed for every value of $n$ : there is an increment $\delta n$ between consecutive data points $(\delta n=40$ for $n<1,200)$. So the onset $n_{0}$ is only accurate up to $\delta n$. This impacts on the goodness of fit since least-squares fitting is known to be sensitive to outliers. Better results are obtained by linearly interpolating the correlation coefficient between consecutive data points and picking the value of $n$ corresponding to the threshold.

Results. Summarizing, we showed that the number of free non-oriented bitangents (which is exactly half the number of oriented bitangents) in a scene consisting of $n$ randomly distributed disjoint unit discs is approximated by

$$
\left(\frac{8.74}{\mu}+2.84-9.59 \mu\right) n-\frac{2,091}{\mu}+9,628-11,895 \mu \text { for } n>\frac{16.77}{\mu}+47.55
$$

where $\mu$ denotes the density of the scene.

The approximation is good in the sense that, in our experiments, for all the densities and all numbers of discs greater than $\frac{16.77}{\mu}+47.55$, the error between the observed and estimated number of bitangents is small. More precisely, this error does not exceed $2 \%$ for densities in the range $[0.05,0.55]$. For smaller densities, the error increases to roughly $10 \%$ for $\mu=0.025$ and $30 \%$ for $\mu=0.0125$. For densities less than or equal to 0.01 , the number of discs in our experiments is 1,200 which is less than the estimated linear onset and we thus do not have a measurement of the error.

Note that even though the $y$-intercept of Equation (2) is not always smaller than the $y$-intercept of the theoretical upper bound of Theorem 1 (as hinted in Figure 7.b), a straightforward computation yields that the estimated number of free bitangents (Eq. (2)) is always less than the upper bound of Theorem 1 for $n \geqslant 1$. (Indeed, if $F(n)$ denotes the upper bound minus the estimated number of bitangents, as a function of $n$, both $F(1)$ and the slope of $F$ are positive for all densities $\mu>0$.)

\subsubsection{Analysis for low densities}

To evaluate the quality of our interpolation for low densities, we ran some specific experiments for density 0.0025 (see Figure 2). We implemented a brute force algorithm for computing the number of bitangents which, compared to 

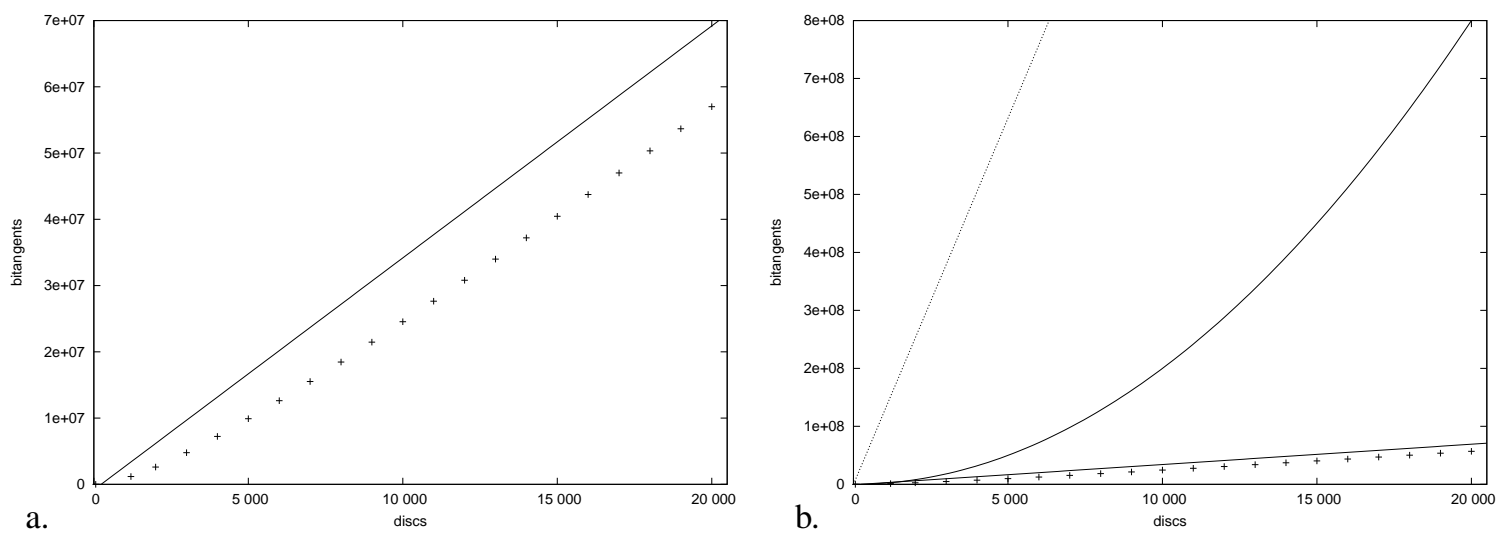

Figure 9: Number of non-oriented bitangents for density 0.0025, and estimate of Eq. (2) for $n>6,755$, with, in (b), the number $4\left(\begin{array}{l}n \\ 2\end{array}\right)$ of possibly obstructed bitangents and the theoretical upper bound of Theorem 1 (in dashed).

Angelier's implementation, is extremely slow but, since it merely counts the bitangents without storing them, uses no memory and therefore allowed us to compute the number of bitangents for rather large numbers of discs. We ran that experiment on random test scenes from 1,000 to 20,000 discs with an increment by one thousand. The entire set of experiments took over 14 days to compute. Figure 9 shows the results of these experiments as well as the interpolated number of bitangents obtained from Equation (2): 3,501 $n-826,846$ for $n>6,755$. As Figure 9.a shows, the slope of the asymptote of the number of bitangents seems well estimated by Eq. (2) but the error on the $y$-intercept is substantial, leading to an error on the number of bitangents decreasing (strictly) from $34.4 \%$ to $17.6 \%$ for $n$ ranging from 7,000 to 20,000. However, as Figure 9.b shows, the estimate is rather accurate when compared to the theoretical upper bound of Theorem 1 or to the number, $4\left(\begin{array}{l}n \\ 2\end{array}\right)$, of possibly obstructed bitangents.

\subsubsection{Analysis for high densities}

The above experimental study focuses on scenes whose density ranges in $[0.0025,0.55]$. Within this density range, we estimated the asymptotic properties of the number of bitangents in terms of the number of discs. We show here that this estimation is likely to be reasonable even for very large densities.

We consider an hexagonal grid as follows; see Figure 10. For any integer $i \geqslant 1$, the grid $\mathcal{G}_{i}$ consists of one central hexagon and $i$ rings of hexagons. We set the distance between the centers of adjacent hexagons to be equal to $2(1+\varepsilon)$. We place one unit disc in each hexagon of the grid and we choose $\varepsilon>0$ small enough so that any pair of discs that are not on the boundary of the grid admit no free outer bitangent. All the centers of the discs in grid $\mathcal{G}_{i}$ are contained in a disc of radius $R_{i}=(1+2 i)(1+\varepsilon)-1$. Let $m_{i}=6 i$ be the number of hexagons in ring $i$. The grid $G_{i}$ contains $n_{i}=1+\sum_{j=1}^{i} m_{j}=1+3 i(i+1)$ hexagons, thus the density of centers in the disc of radius $R_{i}$ is $\mu_{i}=\frac{n_{i}}{R_{i}^{2}}$, a decreasing function of $i$ which tends to $\frac{3}{4(1+\varepsilon)^{2}}$.

The number of non-oriented bitangents in $\mathcal{G}_{i}$ is as follows. Every disc admits 2 inner bitangents with each of its neighboring discs and with no other disc (for $\varepsilon$ sufficiently small); furthermore, all discs have 6 neighboring discs except for $6(i-1)$ discs on the boundary of the grid which have 4 neighbors and 6 discs on the boundary of the grid which have 3 neighbors. Summing, and taking into account that each inner bitangent is counted twice, we get that the number of inner non-oriented bitangents in $\mathcal{G}_{i}$ is $n_{i-1} \cdot 6+6(i-1) \cdot 4+6 \cdot 3=6 i(3 i+1)$. The discs on the boundary of the grid also admit outer bitangents: the number of outer bitangents between the $i+1$ discs on one of the six sides of the hexagonal ring is between $i$ (if the discs are in "convex position") and $\frac{i(i+1)}{2}$ (if the discs are in "concave position"). Hence, the total number $\tau_{i}$ of of non-oriented bitangents in $\mathcal{G}_{i}$ is between $6 i(3 i+2)$ and $3 i(7 i+3)$.

As can be seen, when $i$ is greater than $25, n_{i}$ is larger than 1,951 , the density $\mu_{i}$ lies in $\left(\frac{0.75}{(1+\varepsilon)^{2}}, \frac{0.78}{(1+\varepsilon)^{2}}\right)$ and the ratio $\tau_{i} / n_{i}$ lies in $(5.92,7)$.

For $\varepsilon$ sufficiently small, it is reasonable to believe that any scene of $n_{i}$ unit discs in a disc of radius $R_{i}+1$ has 


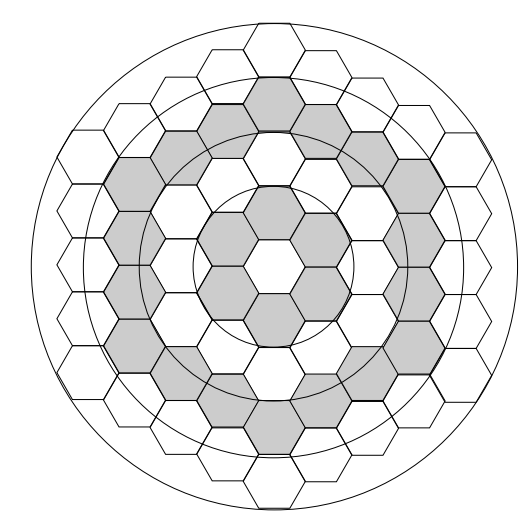

Figure 10: Hexagonal scene model $\left(G_{4}\right)$.

roughly the same number of bitangents because the density is high enough that is seems unlikely that scenes may have substantially different combinatorial characteristics ${ }^{2}$. If this assumption is correct, then the slope of the number of non-oriented bitangents estimated for random scenes should apply. For a density of 0.75, Equation (2) gives an estimated slope of 7.3 instead of some value in $(5.92,7)$ in our analysis. Hence, the estimated slope in Equation (2) is reasonably close to the expected slope of the number of bitangents.

\section{Conclusion}

We have studied the expected size of the $2 \mathrm{D}$ visibility complex of randomly distributed objects in the plane. We proved that the expected asymptotic number of free bitangents among unit discs (or polygons of bounded aspect ratio and similar size) is linear and exhibited bounds in terms of the density of the objects. We also made an experimental assessment of the size of the visibility complex for disjoint random unit discs.

Our experiments give a good idea of the asymptotic behavior of the number of bitangents while our theoretical bound is very rough (see Figure 9.b). Furthermore, the fact that the estimated asymptotic rate of growth is reasonably small in our random setting indicates that the size of the visibility complex might be tractable in practical, real-world applications. As an example, for a reasonable density of $\mu=0.1$ (see Figure 2) and for $n>215$ we can expect $90 n-12,500$ bitangents.

It should be noticed that the visibility complex package [1] we used for our experiments is extremely fast (see Figure 6) especially compared to a brute force algorithm (see Section 4.2.2). However, unlike the brute force approach, the package uses a substantial amount of memory and this prevented us from running experiments for very low density and very large numbers of discs. This situation can be improved by using the antichain feature of the package which, using only $O(n)$ storage, reports the list of free bitangents without storing them in main memory. This feature allows us to compute, with $1 \mathrm{~GB}$ of memory the number of bitangents among up to 3,500 discs in a scene of density 0.0025 compared to 1,500 discs without using the antichain feature.

\section{Acknowledgment}

The authors would like to thank anonymous referees for their valuable comments.

\section{References}

[1] P. Angelier and M. Pocchiola. CGAL-based implementation of visibility complexes. Technical Report ECG-TR-241207-01, Effective Computational Geometry for Curves and Surfaces (ECG), 2003.

\footnotetext{
${ }^{2}$ Note that not much is known on optimal discs packing inside a disc; see [11].
} 
[2] P. Angelier and M. Pocchiola. A sum of squares theorem for visibility complexes and applications. In B. Aronov, S. Basu, J. Pach, and M. Sharir, editors, Discrete and Computational Geometry, volume 25 of Algorithms and Combinatorics, pages 79-139. Springer-Verlag, 2003.

[3] CGAL: Computational Geometry Algorithms Library. http://www.cgal.org.

[4] F. Cho and D. Forsyth. Interactive ray tracing with the visibility complex. Computers and Graphics, 23(5):703-717, 1999. Special issue on Visibility - Techniques and Applications.

[5] O. Devillers, V. Dujmović, H. Everett, X. Goaoc, S. Lazard, H.-S. Na, and S. Petitjean. The expected number of 3D visibility events is linear. SIAM Journal on Computing, 32(6):1586-1620, 2003.

[6] F. Durand. Visibilité tridimensionnelle : étude analytique et applications. PhD thesis, Université Joseph Fourier - Grenoble I, 1999.

[7] F. Durand. A multidisciplinary survey of visibility, 2000. ACM Siggraph course notes, Visibility, Problems, Techniques, and Applications.

[8] F. Durand, G. Drettakis, and C. Puech. The visibility skeleton: a powerful and efficient multi-purpose global visibility tool. Computer Graphics Proceedings, Annual Conference Series, 31:89-100, 1997. Proceedings of Siggraph'97.

[9] F. Durand, G. Drettakis, and C. Puech. The 3D visibility complex. ACM Transactions on Graphics, 21(2):176-206, 2002.

[10] ExpLab - A Tool Set for Computational Experiments. S. Hert, L. Kettner, T. Polzin, G. Schäfer. http://explab. sourceforge.net.

[11] R. L. Graham, B. D. Luboachevsky, K. J. Nurmela, and P. R. J. Östergård. Dense packings of congruent circles in a circle. Discrete Mathematics, 181:139-154, 1998.

[12] N. Holzschuch, F. Sillion, and G. Drettakis. An efficient progressive refinement strategy for hierarchical radiosity. In Proc. of 5th Eurographics Workshop on Rendering, pages 353-357, 1994. Photorealistic Rendering Techniques, Focus on Computer Graphics Series.

[13] K. Kedem, R. Livne, J. Pach, and M. Sharir. On the union of Jordan regions and collision-free translational motion amidst polygonal obstacles. Discrete and Computational Geometry, 1:59-71, 1986.

[14] A. Laurentini. The visual hull concept for silhouette-based image understanding. IEEE Transactions on Pattern Analysis and Machine Intelligence, 16(2):150-162, February 1994.

[15] A. M. Mathai. An Introduction to Geometrical Probability: Distributional Aspects with Applications. Gordon and Breach Sciences Publishers, 1999.

[16] A. Papoulis. Probability, Random Variables, and Stochastic Processes. McGraw-Hill, 1991. 3rd ed.

[17] M. Pellegrini. Ray shooting on triangles in 3-space. Algorithmica, 9:471-494, 1993.

[18] H. Plantinga and C. Dyer. Visibility, occlusion, and the aspect graph. International Journal of Computer Vision, 5(2):137-160, 1990.

[19] M. Pocchiola and G. Vegter. Topologically sweeping visibility complexes via pseudo-triangulations. Discrete and Computational Geometry, 16(4):419-453, 1996. Proceedings of 11th SoCG (ACM Annual Symposium on Computational Geometry), Vancouver, Canada.

[20] M. Pocchiola and G. Vegter. The visibility complex. International Journal of Computational Geometry and Applications, 6(3):1-30, 1996. Proceedings of 9th SoCG (ACM Annual Symposium on Computational Geometry).

[21] L. Santaló. Integral Geometry and Geometric Probability, volume 1 of Encyclopedia of Mathematics and its Applications. Addison-Wesley, 1976. 